\title{
On the Rainbow and Strong Rainbow Coloring of Comb Product Graphs
}

\author{
Dafik ${ }^{1,2, *}$, I.H. Agustin ${ }^{1,3}$, D.A.R. Wardanai ${ }^{1,4}$, E.Y. Kurniawati ${ }^{1,3}$, R. Alfarisi ${ }^{1,5}$ \\ ${ }^{1}$ CGANT, University of Jember, Indonesia \\ ${ }^{2}$ Mathematics Edu. Depart. University of Jember, Indonesia \\ ${ }^{3}$ Mathematics Depart. University of Jember, Indonesia \\ ${ }^{4}$ IKIP PGRI Jember, Indonesia \\ ${ }^{5}$ Department of Elementary School Teacher Education, University of Jember, Indonesia
}

\begin{abstract}
Let $G=(V, E)$ be a simple, nontrivial, finite, connected and undirected graph. Let $c$ be a coloring $c: E(G) \rightarrow\{1,2, \ldots, k\}, k \in N$. A path of the edges of a colored graph is said to be a rainbow path if no two edges on the path have the same color but the adjacent edges may be colored by the same colors. An edge colored graph $G$ is rainbow connected if there exists a rainbow $u-v$ path for every two vertices $u$ and $v$ of $G$. Furthermore, for any two vertices $u$ and $v$ of $G$, a rainbow $u-v$ geodesic in $G$ is a rainbow $u-v$ path of length $d(u, v)$, where $d(u, v)$ is the distance between $u$ and $v$. The graph $G$ is strongly rainbow connected if there exists a rainbow $u-v$ geodesic for any two vertices $u$ and $v$ in $G$. The rainbow and strong rainbow connection numbers of a graph $G$, denoted by $\operatorname{rc}(G)$ and $\operatorname{src}(G)$ respectively, are the minimum number of colors that are needed in order to make $G$ rainbow and strongly rainbow connected, respectively. Some results have shown the lower and upper bound of $r c(G)$ and $\operatorname{src}(G)$, but most of them are not sharp. Thus, finding an exact value of $r c(G)$ and $\operatorname{src}(G)$ are significantly useful. In this paper, we study the exact values of rainbow and strong rainbow connection numbers of comb product graphs.
\end{abstract}

Keywords: rainbow connection; strong rainbow connection; comb product of graphs.

\section{Introduction}

In this paper, we study a graph which is nontrivial, finite, simple, undirected and connected. For all terminology related to the graph elements, we lead reader to see [8]. Recently, a security of delivery system becomes a main issue. Here we consider a graph representation as a network topology. There are many ways to strengthen the security of delivery system concept, such as requiring hamiltonicity, $k$-connectivity, imposing bounds on the diameter, and so on. Motivated by an edge coloring and security of delivery system design, Chartrand et al. [4] in 2008 introduced an interesting way to strengthen the connectivity requirement, namely a rainbow connection of graph.

Let $c$ be a coloring $c: E(G) \rightarrow\{1,2, \ldots, k\}, k \in N$. A path of the edges of a colored graph is said to be a rainbow path if no two edges on the path have the same color but the adjacent edges may be colored by the same colors. An edge colored graph $G$ is rainbow connected if there exists a rainbow $u-v$ path for every two vertices $u$ and $v$ of $G$. Furthermore, for any two vertices $u$ and $v$ of $G$, a rainbow $u-v$ geodesic in $G$ is a rainbow $u-v$ path of length $d(u, v)$, where $d(u, v)$ is the distance between $u$ and $v$. The graph $G$ is strongly rainbow connected if there exists a rainbow $u-v$ geodesic for any two vertices $u$ and $v$ in $G$. The rainbow and strong rainbow connection numbers of a graph $G$, denoted by $r c(G)$ and $\operatorname{src}(G)$ respectively, are the minimum number of colors that are needed in order to make $G$ rainbow and strongly rainbow 
connected, respectively. Some results have shown the lower and upper bound of $r c(G)$ and $\operatorname{src}(G)$, but most of them are not sharp. Determination of the rainbow or strong rainbow connection numbers for any graph are considered to be a complex problem. Suppose an edge coloring of the graph is given. How do we decide whether the colored graphs are rainbow or strong rainbow connected or not? This problem can not be solved in the polynomial times, it is an NP-Complete problem.

There are some results regarded to rainbow coloring described in $[1,2,5,7]$, but the problems are still open since the study has been much focused on special graphs. In [1], Caro, Lev, Roditty, Tuza and Yuster investigated the upper bound of the rainbow connection number of graphs and they proved that if $G$ is a connected graph of order $n$ and with minimum degree $\delta(G) \geq 3$, then $r c(G)<5 n / 6$. Schiermeyer [12] sharped the upper bound by proving that if $G$ is a connected graph of order $n$ with $\delta(G) \geq 3$, then $r c(G)<(3 n-1) / 4$. Chandran et. al. [2] also proved that for every connected graph $G$ of order $n$ and minimum degree $\delta, r_{c}(G) \leq(3 n+1) /$ $\delta+3$. For complete results of upper bounds of rainbow connection number, the readers are referred to [10]. Dafik et.al. [6] studied the rainbow coloring for some graph operations. Agustin, et.al. [9] determined the rainbow $k$-connection number of special graphs and its sharp lower bound.

There are many results regarded to rainbow connection number, but there are less study focusing into strong rainbow connection number. Thus, finding an exact value of $r c(G)$ and $\operatorname{src}(G)$ are useful. In this paper, we study the exact values of rainbow and strong rainbow connection numbers of comb product graphs.

Chandran et al. [2] proved the following useful proposition.

\section{Proposition 1. [2]}

(i) For any connected graph $G$ of size $m$, $\operatorname{diam}(G) \leq r c(G) \leq \operatorname{src}(G) \leq m$, where $\operatorname{diam}(G)$ is the diameter of $G$,

(ii) $r c(G)=1$ if and only if $G$ is complete,

(iii) $r c(G)=m$ if and only if $G$ is a tree of order $m+1$,

(iv) For cycle $C_{n^{\prime}} n \geq 4, r c\left(C_{n}\right)=\operatorname{src}\left(C_{n}\right)=[n / 2\rceil$,

(v) For wheel $W_{n}$ of order $n+1, r c\left(W_{n}\right)=1$ if $n=3$; $r c\left(W_{n}\right)=2$ if $4 \leq n \leq 6 ; r c\left(W_{n}\right)=3$ if $n \geq 7$.

Let $G$ and $H$ be two connected graphs. Let $o$ be a vertex of $H$. The comb product between graphs $G$ and $H$, denoted by $G \triangleright H$, is a graph obtained by taking one copy of $G$ and $|V(G)|$ copies of $H$ and grafting the $i$-th copy of $H$ at the vertex o to the $i$-th vertex of $G$. $|V(G \triangleright H)|=|V(G)| .|V(H)|$ and $|E(G \triangleright H)|=|V(G)| .|E(H)|+|E(G)|$.

In this paper, we study the exact values of rainbow and strong rainbow connection numbers of comb product of special graphs, namely path and cycle $P_{n} \triangleright C_{m^{\prime}}$ path and fan graph $P_{n} \triangleright F_{m^{\prime}}$ and path and triangular book $P_{n} \triangleright B t_{m}$.

\section{Rainbow Coloring}

First, we will show the results on the rainbow coloring of graphs.

Lemma 1. Let $P_{n}$ be a path of order $n$, and $H$ be any connected graph. For comb product of $P$ and $H$ holds $\operatorname{diam}\left(P_{n} \triangleright H\right) \leq r c\left(P_{n} \triangleright H\right) \leq n . r c(\stackrel{n}{H})+n-1$.

Proof. Let $H$ be any graph of order $p$ and size $q$ and $P_{n}$ be a path of order $n$. From the definition of the comb product of $P_{n}$ and $H$ we have that $\left|V\left(P_{n} \triangleright H\right)\right|=p n \quad$ and $\quad\left|E\left(P_{n} \triangleright H\right)\right|=q n+n-1$. From Proposition 1 it follows

$$
\operatorname{rc}\left(P_{n} \triangleright H\right) \geq \operatorname{diam}\left(P_{n} \triangleright H\right) .
$$

It follows that the rainbow connection number of the comb product $P_{n} \triangleright H$ can not be larger than $n$. $r c(H)+n-1$. Thus

$$
r c(P \triangleright H) \leq n . r c(H)+n-1 .
$$

The inequalities (1) and (2) implies the statement. It completes the proof.

Theorem 1. Let $P_{n}$ and $P_{m}$ be paths of order $n$ and $m$, $n, m \geq 2$. The rainbow connection number of the comb product of $P_{n}$ and $P_{m}$ is

$$
r c\left(P_{n} \triangleright P_{m}\right)=n m-1 \text {. }
$$

Proof. The graph $P_{n} \triangleright P_{m}$ is a connected graph with the vertex set $V\left(P_{n} \triangleright P_{m}\right)=\left\{x_{i}: 1 \leq i \leq n\right\} \cup\left\{x_{i, j}: 1 \leq i \leq n\right.$, $1 \leq j \leq m-1\}$ and the edge set $E\left(P_{n} \triangleright P_{m}\right)=\left\{x_{i} x_{i+1}\right.$ : $1 \leq i \leq n-1\} \cup\left\{x_{i} x_{i, 1}: 1 \leq i \leq n\right\} \cup\left\{x_{i, j} x_{i, j+1}: 1 \leq i \leq n, \quad 1 \leq j \leq m^{-}\right.$ 2\}. Hence $\left|V\left(P_{n} \triangleright P_{m}\right)\right|=n m,\left|E\left(P_{n} \triangleright P_{m}\right)\right|=n m-1$ and $\operatorname{diam}\left(P_{n} \triangleright P_{m}\right)=n+2 m-3$.

We will prove that $r c\left(P_{n} \triangleright P_{m}\right) \geq n m-1$, by assuming that $r c\left(P_{n} \triangleright P_{m}\right)<n m-1$. Suppose we need $(n m-2)$ colors to have all paths of $P_{n} \triangleright P_{m}$ as a rainbow path. By definition of comb product, graph $P_{n} \triangleright P_{m}$ has $n$ copies of subgraph $P_{m^{\prime}}$ denoted by $\left(P_{m}\right)_{i}$ for $1 \leq i \leq n$ and one copy of subgraph $P_{n}$ which is called a backbone path (grafting path).

(a) We known that $u-v$ path with $u \in V\left(\left(P_{m}\right)_{k}\right)$ and 
$v \in V\left(\left(P_{m}\right)_{j}\right)$ for $k \neq j, 1 \leq k, j \leq n$ always go through the vertices on $P_{n}$ such that the rainbow edge color in $P_{n}$ differs from the rainbow edge color in $\left(P_{m}\right)_{i}$. Thus, path $P_{n}$ has $n$ - 1 colors.

(b) Given that every vertex $x_{i, j}$ of subgraph $\left(P_{m}\right)_{i}$ to a vertex $x_{i}$ of $P_{n}$ has only one path, namely $x_{i, m-}$ ${ }_{1}-x_{i, m-2}-\ldots-x_{i, 1}-x_{i}$. Thus, each subgraph of path $\left(P_{m}\right)_{i}$ for $1 \leq i \leq n$ has $m-1$ colors.

(c) Since there are at least two same colors of an edge $e \in E\left(\left(P_{m}\right)_{k}\right)$ with an edge $e^{*} \in E\left(\left(P_{m}\right)_{j}\right)$ for $k \neq j$, $1 \leq k, j \leq n, c(e)=c\left(e^{*}\right)$, the rainbow color of subgraph $\left(P_{m}\right)_{i}$ will have $(n-1)(m-1)+(m-2)=n m-n-1$ colors.

(d) We can take a rainbow path between the vertex $u \in V\left(\left(P_{m}\right)_{k}\right)$ and vertex $v \in V\left(\left(P_{m}\right)_{i}\right)$ for $k \neq j$, $1 \leq k, j \leq n$ namely

$$
\begin{aligned}
& \underbrace{x_{i, s}-\cdots-x_{i, 1}}_{V\left(\left(P_{m}\right)_{k}\right)}-x_{1}-\cdots-x_{l}-\underbrace{x_{i, 1}-\cdots-x_{i, r}}_{V\left(\left(P_{m}\right)_{j}\right)} \\
& \approx \underbrace{e}_{E\left(\left(\stackrel{P}{m}_{m}\right)_{k}\right)}-e^{\prime}-\underbrace{e^{*}}_{E\left(\left(P_{m}\right)_{j}\right)}
\end{aligned}
$$

for $1 \leq s, r \leq m-1$ and $1 \leq l \leq n$. Thus, there will be at least two edges in the rainbow path $u-v$ with $u \in V\left(\left(P_{m}\right)_{k}\right)$ and $v \in V\left(\left(P_{m}\right)_{i}\right)$ having the same colors, namely $c(e)=c\left(e^{*}\right)$. It is a contradiction. Thus, from points (b) and (c), we get $n m-n-1+n-1=n m-2$ colors but it implies that there are at least two edges that have the same color on the rainbow path based on the point (d). Thus, we need at least $n m-1$ colors to color a path of graph $P_{n} \triangleright P_{m}$ such that all its paths are a rainbow path. We can conclude that the lower bound of the rainbow connection number of $P_{n} \triangleright P_{m}$ is $r c\left(P_{n} \triangleright P_{m}\right) \geq n m-1$. Furthermore, we will prove that the upper bound of the rainbow connection number of $P_{n} \triangleright P_{m}$ is $r c\left(P_{n} \triangleright P_{m}\right) \leq n m-1$. Define the edge coloring $c: E\left(P_{n} \triangleright P_{m}\right)^{n} \rightarrow\{1,2, \ldots, n m$ $1\}$ as follows

$$
c(e)=\left\{\begin{array}{c}
i, \quad e=x_{i} x_{i+1} ; 1 \leq i \leq n-1 \\
n+(i-1)(m-1), \quad e=x_{i} x_{i, j} ; 1 \leq i \leq n, j=1 \\
n+(i-1)(m-1)+j, \quad e=x_{i, j} x_{i, j+1} ; 1 \leq i \leq n, \\
1 \leq j \leq m-2 .
\end{array}\right.
$$

It gives a rainbow path from any vertex to other vertices on edge coloring $c: E\left(P_{n} \triangleright P_{m}\right) \rightarrow\{1,2, \ldots$ $, n m-1\}$. Thus, the upper bound of rainbow connection number of $P_{n} \triangleright P_{m}$ is $r_{c}\left(P_{n} \triangleright P_{m}\right) \leq n m-1$. It concludes that $\operatorname{rc}\left(P_{n} \triangleright P_{m}\right)=n m-1$.

Theorem 2. Let $P_{n}$ be a path of order $n, n \geq 2$, and $K_{m}$

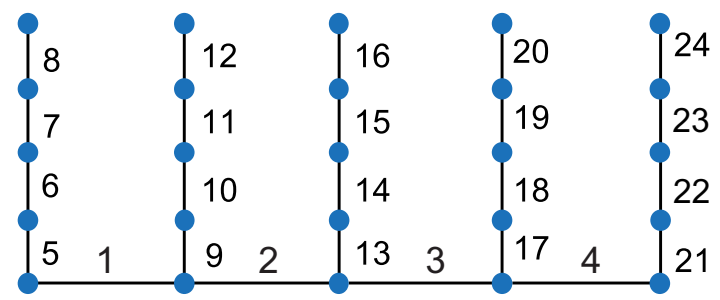

Fig. 1: Example of rainbow connection of $P_{5} \triangleright P_{5}$.

be a complete graph of order $m, m \geq 3$. The rainbow connection number of the comb product between $P_{n}$ and $K_{m}$ is

$$
\operatorname{rc}\left(P_{n} \triangleright K_{m}\right)=2 n-1 .
$$

Proof. The graph $P_{n} \triangleright K_{m}$ is a connected graph with the vertex set $V\left(P_{n} \triangleright K_{m}\right)=\left\{x_{i, j} \cdot 1 \leq i \leq n, 1 \leq i \leq m\right\}$ and the edge set $E\left(P_{n} \triangleright K_{m}\right)=\left\{x_{i, 1} x_{i+1,1}: 1 \leq i \leq n-1\right\}$ $\cup \quad\left\{x_{i, j} x_{i, j+k}: 1 \leq i \leq n, 1 \leq j \leq m-1,1 \leq k \leq m-j\right\}$. Hence $\left|V\left(P_{n} \triangleright K_{m}\right)\right|=n m, \quad\left|E\left(P_{n} \triangleright K_{m}\right)\right|=n m(m-1) / 2+n-1 \quad$ and $\operatorname{diam}\left(P_{n} \triangleright K_{m}\right)=n+1$.

We will prove that $r c\left(P_{n} \triangleright K_{m}\right) \geq 2 n$-1, by assuming that $r_{c}\left(P_{n} \triangleright K_{m}\right)<2 n-1$. Suppose we need $(2 n-2)$ colors to have all paths of $P_{n} \triangleright K_{m}$ as a rainbow path. By definition of comb product $P_{n} \triangleright K_{m^{\prime}}$, it will have $n$ copies of subgraph $K_{m^{\prime}}$ denoted by $\left(K_{m}\right)_{i}$ for $1 \leq i \leq n$ and one subgraph $P_{n}$ which is called a backbone path (grafting path).

(a) The path $u-v$ with $u \in V\left(\left(K_{m}\right)_{k}\right)$ and $v \in V\left(\left(K_{m}\right)_{j}\right)$ for $k \neq j, 1 \leq k, j \leq n$, always go through the vertices on $P_{n}$ such that the rainbow edge color in $P_{n}$ differs with that edge rainbow path in $\left(K_{m}\right)_{i}$. Thus, path $P_{n}$ has $n$ - 1 rainbow colors.

(b) Each path from $x_{i, j}$ of subgraph $\left(K_{m}\right)_{i}$ to a vertex $x_{i, 1,}$ of $P_{n}$ has only one path, namely $x_{i, j}$ $x_{i, 1}$ (since the diameter of $\left(K_{m}\right)_{i}$ is 1$)$. Thus, each complete subgraph $\left(K_{m}\right)_{i}$ for $1 \leq i \leq n$ has one color.

(c) Since there are at least two same colors of an edge $e \in E\left(\left(K_{m}\right)_{k}\right)$ with an edge $e^{*} \in E\left(\left(K_{m}\right)_{j}\right)$ for $k \neq j$, $1 \leq k, j \leq n, c(e)=c\left(e^{*}\right)$, the rainbow color of subgraph $\left(K_{m}\right)_{i}$ will have $n-1$ colors.

(d) Suppose we take the rainbow path of vertex $u \in V\left(\left(K_{m}\right)_{k}\right)$ and vertex $v \in V\left(\left(K_{m}\right)_{j}\right)$ for $k \neq j_{,} 1 \leq k_{i} j \leq n$ namely

$$
\begin{aligned}
& \underbrace{x_{k, s}}_{V\left(\left(K_{m}\right)_{k}\right)}-x_{k, 1}-\cdots-x_{j, 1}-\underbrace{x_{j, r}}_{V\left(\left(K_{m}\right)_{j}\right)}
\end{aligned}
$$

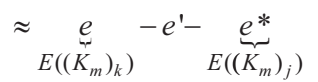

for $1 \leq s, r \leq m$ and $1 \leq k, j \leq n$, thus there are at least two edges of rainbow path $u-v$ with $u \in V\left(\left(K_{m}\right)_{k}\right)$ 
and $v \in V\left(\left(K_{m}\right)_{i}\right)$ having the same colors, namely $c(e)=c\left(e^{*}\right)$. It is a contradiction.

Thus, by points (b) and (c), we get $n-1+n-1=$ $2 n-2$ colors, but it implies that there are at least two edges having the same color on its rainbow path based on the point (d). Thus, we can not avoid to have $2 n$ - 1 colors to color a path of graph $P_{n} \triangleright K_{m}$ such that all its paths are a rainbow path. We can conclude that the lower bound of the rainbow connection number of $P_{n} \triangleright K_{m}$ is $r c\left(P_{n} \triangleright K_{m}\right) \geq 2 n-1$. Furthermore, we will prove that the upper bound of the rainbow connection number of $P_{n} \triangleright K_{m}$ is $r c\left(P_{n} \triangleright K_{m}\right) \leq 2 n-1$. Define the edge coloring $c$ : $E\left(P_{n} \triangleright K_{m}\right) \rightarrow\{1,2, \ldots, 2 n-1\}$ as follows

$c(e)=\left\{\begin{array}{c}i, \quad e=x_{i, 1} x_{i+1,1} ; 1 \leq i \leq n-1 \\ n-1+i, \quad e=x_{i, j} x_{i, j+k} ; 1 \leq i \leq n, 1 \leq j \leq m-1, \\ 1 \leq k \leq m-j .\end{array}\right.$

It gives a rainbow path from any vertex to other vertices on edge coloring $c: E\left(P_{n} \triangleright K_{m}\right)$ $\rightarrow\{1,2, \ldots, 2 n-1\}$. Thus, the upper bound of rainbow connection number of $P_{n} \triangleright K_{m}$ is $r c\left(P_{n} \triangleright K_{m}\right) \leq 2 n$-1. It concludes that $r c\left(P_{n} \triangleright P_{m}^{n}\right)=2 n-1$.

Theorem 3. Let $P_{n}$ be a path of order $n, n \geq 2$, and $S_{m}$ be a star of order $m+1, m \geq 1$. The rainbow connection number of the comb product of $P_{n}$ and $S_{m}$ is

$$
r c\left(P_{n} \triangleright S_{m}\right)=m n+n-1 \text {. }
$$

Proof. The graph $P_{n} \triangleright S_{m}$ is a connected graph with the vertex set $V\left(P_{n} \triangleright S_{m}\right)=\left\{x_{i}: 1 \leq i \leq n\right\} \cup\left\{x_{i, j}: 1 \leq i \leq n\right.$, $1 \leq j \leq m\}$ and the edge set $E\left(P_{n} \triangleright S_{m}\right)=\left\{x_{i} x_{i+1}: 1 \leq i \leq n-1\right\}$ $\cup\left\{x_{i} x_{i, j}: 1 \leq i \leq n, 1 \leq j \leq m\right\}$. Hence $\left|V\left(P_{n} \triangleright S_{m}\right)\right|=n m+n$, $\left|E\left(P_{n} \triangleright S_{m}\right)\right|=n m+n-1$ and $\operatorname{diam}\left(P_{n} \triangleright S_{m}\right)=n+1$.

The grafting vertex of star $S_{m}$ is a vertex of degree one or pendant vertex. We will prove the lower bound of $r c\left(P_{n} \triangleright S_{m}\right) \geq m n+n-1$, by assuming that $r_{c}\left(P_{n} \triangleright S_{m}\right)<m n+n-1$. Suppose we need $(m n+n-$ 2) colors to have all paths of $P_{n} \triangleright S_{m}$ as a rainbow path. Based on the definition of the comb product $P_{n} \triangleright S_{m^{\prime}}$ it will has $n$ copies of subgraph $S_{m^{\prime}}$ denoted by $\left(S_{m}\right)_{i}$ for $1 \leq i \leq n$ and one subgraph $P_{n}$ which is called a backbone path (grafting path).

(a) We know that $u-v$ with $u \in V\left(\left(S_{m}\right)_{k}\right)$ and $v \in V\left(\left(S_{m}\right)_{j}\right)$ for $k \neq j, 1 \leq k j \leq n$ always go through the vertex in path $P_{n}$ thus the edge rainbow color of path $P_{n}$ differs the edge rainbow color of path in $\left(S_{m}\right)_{i}$. Thus, path $P_{n}$ has $n-1$ colors to be all rainbow path.

(b) Since the diameter of $\left(S_{m}\right)_{i}$ is 2, each path from vertex $x_{i, j}$ of subgraph $\left(S_{m}\right)_{i}$ to vertex $x_{i, 1}$ in $P_{n}$ have only one path namely $x_{i, k}-x_{i, 1}$. Thus, each subgraph of star $\left(S_{m}\right)_{i}$ for $1 \leq i \leq n$ has $m$ colors which induce a rainbow coloring of star graph.

(c) Since there are at least two same colors of an edge $e \in E\left(\left(S_{m}\right)_{k}\right)$ with an edge $e^{*} \in E\left(\left(S_{m}\right)_{j}\right)$ for $k \neq j$, $1 \leq k, j \leq n, c(e)=c\left(e^{*}\right)$. The rainbow color of $\left(S_{m}\right)_{i}$ will have $m(n-1)+m-1=n m-1$ colors.

(d) Suppose we take the rainbow path of vertex $u \in V\left(\left(S_{m}\right)_{k}\right)$ and $v \in V\left(\left(S_{m}\right)_{i}\right)$ for $k \neq j, 1 \leq k, j \leq n$ namely

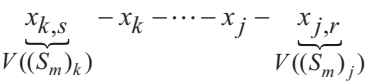

$$
\begin{aligned}
& \approx \underset{E\left(\left(S_{m}\right)_{k}\right)}{e}-e^{\prime}-\underbrace{e^{*}}_{E\left(\left(S_{m}\right)_{j}\right)}
\end{aligned}
$$

for $1 \leq s, r \leq m$ and $1 \leq k, j \leq n$, there are at least two edges of rainbow path $u-v$ with $u \in V\left(\left(S_{m}\right)_{k}\right)$ and $v \in V\left(\left(S_{m}\right)_{i}\right)$ having the same colors, namely $c(e)=c\left(e^{*}\right)$. It is a contradiction.

Based on the points (b) and (c) we have $\mathrm{nm}^{-}$ $1+n-1=n m+n-2$ colors but it implies that there are at least two edges having the same color in the rainbow path based on the point (d). Thus, we can not avoid to have at least $n m+n-1$ colors to make all paths of $P_{n} \triangleright S_{m}$ as a rainbow path. We conlude that the lower bound of the rainbow connection number of $P_{n} \triangleright S_{m}$ is $r c\left(P_{n} \triangleright S_{m}\right) \geq n m+n-1$. Furthermore, we will prove the upper bound of the rainbow connection number of $P_{n} \triangleright S_{m}$ that is $r c\left(P_{n} \triangleright S_{m}\right) \leq n m+n-1$. Define the edge coloring $c$ : $E\left(P_{n} \triangleright S_{m}\right) \rightarrow\{1,2,3, \ldots, n m+n-1\}$ as follows.

$$
c(e)=\left\{\begin{array}{l}
i, \quad e=x_{i} x_{i+1} ; 1 \leq i \leq n \\
n j-1+i, \quad e=x_{i} x_{i, j} ; 1 \leq i \leq n, 1 \leq j \leq m .
\end{array}\right.
$$

It gives a rainbow path from any vertex to other vertices on edge coloring $c: E\left(P_{n} \triangleright S_{m}\right) \rightarrow\{1,2, \ldots$, $n m+n-1\}$. Thus, the upper bound of rainbow connection number of $P_{n} \triangleright S_{m}$ is $r c\left(P_{n} \triangleright S_{m}\right) \leq n m+n-1$. Therefore, $r c\left(P_{n} \triangleright S_{m}\right)=n m+n-1$.

Theorem 4. Let $P_{n}$ be a path of order $n, n \geq 2$, and $C_{m}$ be a cycle of order $m, m \geq 3$. The rainbow connection number of the comb product of $P_{n}$ and $C_{m}$ is

$$
\operatorname{rc}\left(P_{n} \triangleright C_{m}\right)=m+n-1 \text {. }
$$

Proof. The graph $P_{n} \triangleright C_{m}$ is a connected graph with the vertex set $\left.V\left(P_{n} \triangleright C_{m}\right)=x_{i, j}: 1 \leq i \leq n, 1 \leq j \leq m\right\}$ and the edge set $E\left(P_{n}^{n} \triangleright C_{m}^{m}\right)=\left\{x_{i, 1}^{i, j} x_{i+1,1}: 1 \leq i \leq n-1\right\}$ $\cup\left\{x_{i, j} x_{i, j+1}, x_{i, m} x_{i, 1}: 1 \leq i \leq n, \quad 1 \leq j \leq m-1\right\}$. Hence we 
get $\left|V\left(P_{n} \triangleright C_{m}\right)\right|=n m, \quad\left|E\left(P_{n} \triangleright C_{m}\right)\right|=n m+n-1 \quad$ and $\operatorname{diam}\left(P_{n} \triangleright C_{m}\right)=2\lfloor m / 2\rfloor+n-1$.

Based on Proposition 1, we have that $\operatorname{rc}_{c}\left(P_{n} \triangleright C_{m}\right)$ $\geq \operatorname{diam}\left(P_{n} \triangleright C_{m}\right)=2\lfloor m / 2\rfloor+n-1$. If $m$ is even then $2\lfloor m / 2\rfloor+n-1 .=2(m / 2)+n-1=m+n-1$ and if $m$ is odd then $2\lfloor m / 2\rfloor+n-1=2((m-1) / 2)+n-1=m+n-2$. Hence $\operatorname{rc}\left(P_{n} \triangleright C_{m}\right) \geq \max \{m+n-1, m+n-2\}=m+n-1$.

In order to show that $\operatorname{rc}\left(P_{n} \triangleright C_{m}\right) \leq m+n-1$, we define an edge coloring $c: E\left(P_{n} \triangleright C_{m}\right) \rightarrow\{1,2, \ldots, n+m$ $1\}$ of $P_{n} \triangleright C_{m}$ in the following way:

$$
c(e)=\left\{\begin{array}{l}
i, \quad e=x_{i, 1} x_{i+1,1} ; 1 \leq i \leq n-1 \\
n-1+j, \quad e=x_{i, j} x_{i, j+1} ; 1 \leq i \leq n, 1 \leq j \leq m-1 \\
n-1+m, \quad e=x_{i, 1} x_{i, m} ; 1 \leq i \leq n .
\end{array}\right.
$$

The edge coloring gives a rainbow path between every two vertices of $P_{n} \triangleright C_{m}$. Therefore, $r c\left(P_{n} \triangleright C_{m}\right) \leq m+n-1$. Hence $r c\left(P_{n} \triangleright C_{m}\right)=m+n-1$ and it completes the proof.

As an illustration, we give Figure 2.

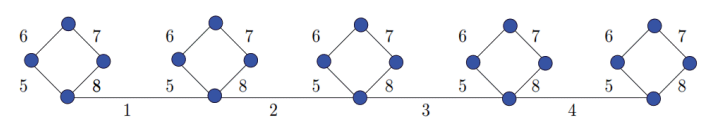

Fig. 2: Example of rainbow connection of $P_{5} \triangleright C_{4}$.

Theorem 5. Let $P_{n}$ be a path of order $n, n \geq 2$, and $F_{m}$ be a fan of order $m+1, m \geq 2$. The rainbow connection number of the comb product between $P_{n}$ and $F_{m}$ is $r c\left(P_{n} \triangleright F_{m}\right) \leq m+n-1$.

Proof. Let o be the central vertex of the fan $F_{m}$. Then the graph $P_{n} \triangleright F_{m}$ is a connected graph with the vertex set $V^{n}\left(P_{n} \triangleright F_{m}\right)=\left\{x_{i^{i}} 1 \leq i \leq n\right\} \cup\left\{y_{i, j}: 1 \leq i \leq n\right.$, $1 \leq j \leq m\}$ and the edge set $E\left(P_{n} \triangleright F_{m}\right)=\left\{x_{i} x_{i+1}: 1 \leq i \leq n\right.$ $1\} \cup\left\{x_{i} y_{i, j}: 1 \leq i \leq n, 1 \leq j \leq m\right\} \cup\left\{y_{i, j} y_{i, j+1}: 1 \leq i \leq n, 1 \leq j \leq m-\right.$ 1\}. Hence $\left|V\left(P_{n} \triangleright F_{m}\right)\right|=n(m+1),\left|E\left(P_{n} \triangleright F_{m}\right)\right|=2 n m-1$ and $\operatorname{diam}\left(P_{n} \triangleright F_{m}\right)=n+1$.

Now, we prove that $r c\left(P_{n} \triangleright F_{m}\right) \leq m+n-1$ by defining an edge coloring $c$ as follows:

$$
c(e)=\left\{\begin{array}{l}
i, \quad e=x_{i} x_{i+1} ; 1 \leq i \leq n-1 \\
n-1+j, \quad e=x_{i} y_{i, j} ; 1 \leq i \leq n, 1 \leq j \leq m \\
n+m-1-j, \quad e=y_{i, j} y_{i, j+1} ; 1 \leq i \leq n, 1 \leq j \leq m-1 .
\end{array}\right.
$$

It is easy to see that the edge coloring $c: E\left(P_{n} \triangleright F_{m}\right)$ $\rightarrow\{1,2, \ldots, m+n-1\}$ is the desired coloring with at most $m+n-1$ colors. It proves that $r c\left(P_{n} \triangleright F_{m}\right) \leq m+n-1$.

The corona product of a graph $G$ with a graph $H$, denoted by $G \odot H$, is a graph obtained by taking one copy of an $n$-vertex graph $G$ and $n$ copies $H_{1}, H_{2}, \ldots, H_{n}$ of $H$ and then joining the $i$-th vertex of the graph $G$ to every vertex in $H_{i}$. The graph $B t_{m}$ $\cong P_{1} \odot S_{m}$ where $S_{m}$ is the star of order $m+1$, we call the triangular book graph.

Theorem 6. Let $P_{n}$ be a path of order $n, n \geq 2$, and $B t_{m}$ be a triangular book graph of order $m+2, m \geq 1$. The rainbow connection number of the comb product between $P_{n}$ and $B t_{m}$ is

$$
r c\left(P_{n} \triangleright B t_{m}\right)=n+2 .
$$

Proof. Let $o$ be the vertex of $P$ in the triangular book $B t_{m}$. The graph $P_{n} \triangleright B t_{m}$ is a connected graph with the vertex set $V\left(P_{n} \triangleright B t_{m}\right)=\left\{a_{i^{\prime}} b_{i}: 1 \leq i \leq n\right\} \cup\left\{y_{i, j}: 1 \leq i \leq n\right.$, $1 \leq j \leq m\}$ and the edge set $E\left(P_{n} \triangleright B t_{m}\right)=\left\{a_{i} a_{i+1}: 1 \leq i \leq n\right.$ $1\} \cup\left\{b_{i} y_{i, j}: 1 \leq i \leq n, 1 \leq j \leq m\right\} \cup\left\{a_{i} y_{i, j}: 1 \leq i \leq n, 1 \leq j \leq m\right\}$ $\cup\left\{a_{i} b_{i}: \quad 1 \leq i \leq n\right\}$. Hence $\left|V\left(P_{n} \triangleright B t_{m}\right)\right|=n(m+2)$, $\left|E\left(P_{n} \triangleright B t_{m}\right)\right|=2 n(m+1)-1$ and $\operatorname{diam}\left(P_{n} \triangleright B t_{m}\right)=n+1$.

Based on Proposition 1, we have

$$
\operatorname{rc}\left(P_{n} \triangleright B t_{m}\right) \geq \operatorname{diam}\left(P_{n} \triangleright B t_{m}\right)=n+1 .
$$

Now, we define an edge coloring $c$ of the graph $P_{n} \triangleright B t_{m}$ as follows:

$c(e)=\left\{\begin{array}{l}i, \quad e=a_{i} a_{i+1} ; 1 \leq i \leq n-1 \\ n, \quad e=a_{i} y_{i, j} ; 1 \leq i \leq n, 1 \leq j \leq m \\ n+2, \quad e=b_{i} y_{i, j} ; 1 \leq i \leq n, 1 \leq j \leq m \\ n+1, \quad e=a_{i} b_{i} ; 1 \leq i \leq n .\end{array}\right.$

It is easy to see that the graph $P_{n} \triangleright B t_{m}$ is rainbow connected under the edge coloring c: $E\left(P_{n} \triangleright B t_{m}\right)$ $\rightarrow\{1,2, \ldots, n+2\}$. Therefore, $r c\left(P_{n} \triangleright B t_{m}\right) \leq n+2$.

Now, we suppose that $\operatorname{rc}\left(P_{n} \triangleright B t_{m}\right)=n+1$. However, there is the path between the vertices $y_{1, j}$ and $y_{n, j^{\prime}} 1 \leq j \leq m$, that is not rainbow path because at least $n+2$ colors are necessary. This proves that $\operatorname{rc}\left(P_{n} \triangleright B t_{m}\right)=n+2$.

As an illustration, we give Figure 3.

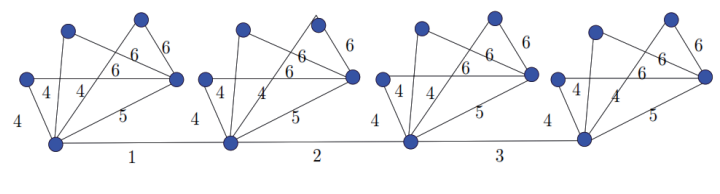

Fig. 3: Example of rainbow connection of $P_{4} \triangleright B t_{3}$.

\section{Strong Rainbow Coloring}

Secondly, we will show the results on the strong rainbow coloring of graphs. 
Lemma 2. Let $P_{n}$ be a path of order $n$, and $H$ be any connected graph. For comb product of $P_{n}$ and $H$ holds $\operatorname{diam}\left(P_{n} \triangleright H\right) \leq \operatorname{src}\left(P_{n} \triangleright H\right) \leq n . \operatorname{src}(H)+n-1$.

Proof. Let $H$ be any graph of order $p$ and size $q$ and $P_{n}$ be a path of order $n$. From Proposition 1 it follows

$$
\operatorname{src}\left(P_{n} \triangleright H\right) \geq \operatorname{diam}\left(P_{n} \triangleright H\right) .
$$

Based on the definition of the comb product $P_{n} \triangleright H$, the graph $H$ is copied $n$ times, and the graph $P_{n} \triangleright H$ is strongly rainbow connected if there exists a rainbow $u-v$ geodesic for any two vertices $u$ and $v$ in $P_{n} \triangleright H$. Thus we have the upper bound for the strong rainbow connection number as follows

$$
\operatorname{src}\left(P_{n} \triangleright H\right) \leq n . \operatorname{src}(H)+\operatorname{src}\left(P_{n}\right)=n . \operatorname{src}(H)+n-1 .
$$

The inequalities (3) and (4) imply that $\operatorname{diam}\left(P_{n} \triangleright H\right)$ $\leq \operatorname{src}\left(P_{n} \triangleright H\right) \leq n . \operatorname{src}(H)+n$-1. It completes the proof.

Theorem 7. Let $P_{n}$ be a path of order $n, n \geq 2$, and $C_{m}$ be a cycle of order $m, m \geq 3$. The strong rainbow connection number of the comb product between $P_{n}$ and $C_{m}$ is

$$
\operatorname{src}\left(P_{n} \triangleright C_{m}\right) \leqslant 2\left\lfloor\frac{m}{2}\right\rfloor+n-1 .
$$

Proof. According to Proposition 1 we get

$$
\operatorname{src}\left(P_{n} \triangleright C_{m}\right) \geq \operatorname{diam}\left(P_{n} \triangleright C_{m}\right)=2\left\lfloor\frac{m}{2}\right\rfloor+n-1 .
$$

With respect to Lemma 2, we have

$$
\begin{aligned}
\operatorname{diam}\left(P_{n} \triangleright C_{m}\right) & \leq \operatorname{src}\left(P_{n} \triangleright C_{m}\right) \leq n . \operatorname{src}\left(C_{m}\right)+n-1 \\
& =2\left\lfloor\frac{m}{2}\right\rfloor+n-1 .
\end{aligned}
$$

Now, we define an edge coloring $c$ of the graph $P_{n} \triangleright C_{m}$ in the following way:

$$
c(e)=\left\{\begin{array}{l}
i, \quad e=x_{i, 1} x_{i+1,1} ; 1 \leq i \leq n-1 \\
n-1+j+(i-1)\lfloor m / 2\rfloor, \\
\quad e=x_{i, j} x_{i, j+1} ; 1 \leq i \leq n, 1 \leq j \leq\lfloor m / 2\rfloor \\
n-1+j-\lfloor m / 2\rfloor+(i-1)\lfloor m / 2\rfloor, \\
\quad \quad e=x_{i, j} x_{i, j+1} ; 1 \leq i \leq n,\lfloor m / 2\rfloor+1 \leq j \leq m-1 \\
n-1+i\lfloor m / 2\rfloor, \quad e=x_{i, 1} x_{i, m} ; 1 \leq i \leq n \\
1, \quad e=x_{i,\lfloor m / 2\rfloor} x_{i,\lfloor m / 2\rfloor+1} ; 1 \leq i \leq n .
\end{array}\right.
$$

It is not difficult to see that the graph $P_{n} \triangleright C_{m}$ is the strong rainbow connected under the edge coloring $c: E\left(P_{n} \triangleright C_{m}\right) \rightarrow\{1,2, \ldots, n\lfloor m / 2\rfloor+n-1\}$. It proves that $\operatorname{src}\left(P_{n} \triangleright C_{m}\right) \leq n\lfloor m / 2\rfloor+n-1$.

Figure 4 gives an example of strong rainbow connection of $P_{5} \triangleright C_{4}$.

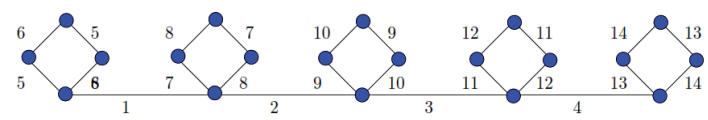

Fig. 4: Example of rainbow connection of $P_{5} \triangleright C_{4}$.

Theorem 8. Let $P_{n}$ be a path of order $n, n \geq 2$, and $F_{m}$ be a fan of order $m+1, m \geq 2$. The strong rainbow connection number of the comb product between $P_{n}$ and $F_{m}$ is

$$
\operatorname{src}\left(P_{n} \triangleright F_{m}\right) \leq m n+n-1 .
$$

Proof. Define an edge coloring $c$ of the graph $P_{n} \triangleright F_{m}$ in the following way:

$c(e)=\left\{\begin{array}{l}i, \quad e=x_{i} x_{i+1} ; 1 \leq i \leq n-1 \\ n-1+j+(i-1) m, \quad e=x_{i} y_{i, j} ; 1 \leq i \leq n, 1 \leq j \leq m \\ 1, \quad e=y_{i, j} y_{i, j+1} ; 1 \leq i \leq n, 1 \leq j \leq m-1 .\end{array}\right.$

We can see that the graph $P_{n} \triangleright F_{m}$ is rainbow connected under the previous edge coloring $c$ which uses at most $m n+n-1$ colors. Thus, the existence of the coloring $c: E\left(P_{n} \triangleright F_{m}\right) \rightarrow\{1,2, \ldots$, $m n+n-1\}$ proves that $\operatorname{src}\left(P_{n} \triangleright F_{m}\right) \leq m n+n-1$.

An example of strong rainbow connection of $P_{n} \triangleright F_{m}$ is depicted in Figure 5.

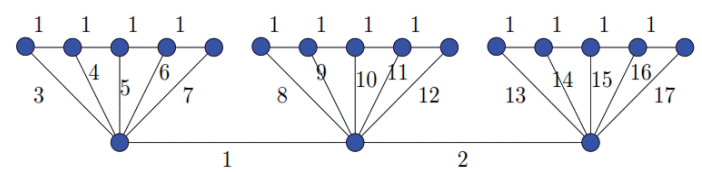

Fig. 5: Example of rainbow connection of $P_{3} \triangleright F_{5}$

Theorem 9. Let $P_{n}$ be a path of order $n, n \geq 2$, and $B t_{m}$ be a triangular book graph of order $m+2, m \geq 1$. The strong rainbow connection number of the comb product between $P_{n}$ and $B t_{m}$ is

$$
\operatorname{src}\left(P_{n} \triangleright B t_{m}\right) \stackrel{m}{\leqslant} m n+n-1 .
$$

Proof. Let us define an edge coloring $c$ : $E\left(P_{n} \triangleright B t_{m}\right)$ $\rightarrow\{1,2, \ldots, m n+n-1\}$ as follows: 


$$
c(e)=\left\{\begin{array}{l}
i, \quad e=a_{i} a_{i+1} ; 1 \leq i \leq n-1 \\
n+m(i-1)+j-1, \quad e=a_{i} y_{i, j} ; 1 \leq i \leq n, 1 \leq j \leq m \\
n+m(i-1)+j-1 \quad e=b_{i} y_{i, j} ; 1 \leq i \leq n, 1 \leq j \leq m \\
n+m(i-1), \quad e=a_{i} b_{i} ; 1 \leq i \leq n .
\end{array}\right.
$$

It is not difficult to verify that the edge coloring $c$ gives a rainbow $u^{-v}$ geodesic for every two vertices $u$ and $v$ of triangular book $P_{n} \triangleright B t_{m}$. Since the coloring $c$ uses at most $n m+n-1$ colors then $\operatorname{src}\left(P_{n} \triangleright B t_{m}\right) \leq n m+n-1$.

As an illustration, we give Figure 6.

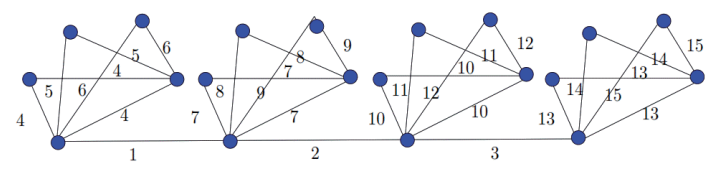

Fig. 6: Example of rainbow connection of $P_{4} \triangleright B t_{3}$.

\section{Concluding Remarks}

In this paper, we have determined the exact values of the rainbow connection number of the comb product between a path $P_{n}$ and a cycle $C_{m^{\prime}}$ and between a path $P_{n}$ and a triangular book graph $B t_{m}$. We obtained only the upper bound of the rainbow connection number of the comb product between path $P_{n}$ and fan $F_{m}$. The rest, for the strong rainbow connection number of $P_{n} \triangleright C_{m^{\prime}}$ $P_{n} \triangleright F_{m}$ and $P_{n} \triangleright B t_{m}$ we have obtained only the upper bounds. Thus we propose the following open problem: "Find the lower bound of the rainbow or strong rainbow connection number of $P_{n} \triangleright C_{m^{\prime}} P_{n} \triangleright F_{m}$ and $P_{n} \triangleright B t_{m}{ }_{m}^{\prime}$.

\section{Acknowledgments \\ We gratefully acknowledge the support from DP2M research grant FUNDAMENTAL and CGANT - University of Jember of year 2017.}

\section{References and Notes}

[1] Caro, Y., Lev, A., Roditty, Y., Tuza, Z., Yuster, R. (2008). On rainbow connection, Electron. J. Combin. 15, R57.

[2] Chandran, L.S., Das, A., Rajendraprasad, D., Varma, N.M. (2010). Rainbow connection number and connected dominating sets, Arxiv preprint arXiv:1010.2296v1 [math.CO].

[3] Chakraborty, S., Fischer, E., Matsliah, A., Yuster, R. (2009). Hardness and algorithms for rainbow connectivity, 26th International Symposium on Theoretical Aspects of Computer Science STACS 2009, p. 243-254.
[4] Chartrand, G., Johns, G.L., McKeon, K.A., Zhang, P. (2008). Rainbow connection in graphs, Math. Bohem. 133, p. 85-98.

[5] Chartrand, G., Johns, G.L., McKeon, K.A., Zhang, P. (2009). The rainbow connectivity of a graph, Networks. 54(2), p. 75-81.

[6] Dafik, Agustin, I. H., Fajariyato, A., Alfarisi, R. (2016). On the rainbow coloring for some graph operations. AIP Conference Proceedings, 1707(1), 020004.

[7] Formanowicz, P., Tana, K. (2012). A Survey Of Graph Coloring - Its Types, Methods And Applications, Foundations of Computing and Decision Sciences. 37.

[8] Gross, J.L., Yellen, J., Zhang, P. (2014). Handbook of Graph Theory, Second Edition, CRC Press, Taylor and Francis Group.

[9] Agustin, I.H., Dafik, Waspodo, G.A., Alfarisi, R. (2017). On rainbow $k$-connection number of special graphs and it's sharp lower bound, IOP Conf. Series: Journal of Physics: Conf. Series $855,012003$.

[10] Li, X., Sun, Y. (2011). Rainbow connections of graphs - A survey, arXiv:1101.5747v2 [math.CO].

[11] Li, X., Sun, Y. (2010). Characterize graphs with rainbow connection number $m-2$ and rainbow connection numbers of some graph operations. preprint.

[12] Schiermeyer, I. (2009). Rainbow connection in graphs with minimum degree three, IWOCA 2009, LNCS 5874. 